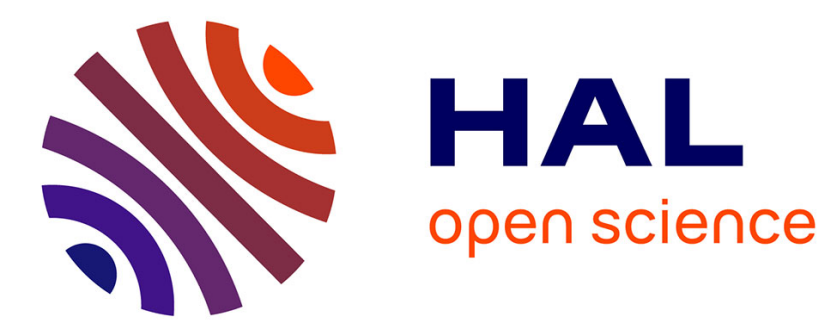

\title{
Power sums of Hecke eigenvalues of Maass cusp forms
} Jie $\mathrm{Wu}$, Zhao Xu

\section{To cite this version:}

Jie $\mathrm{Wu}$, Zhao Xu. Power sums of Hecke eigenvalues of Maass cusp forms. Ramanujan Journal (The), 2015, 36 (3), pp.439-453. 10.1007/s11139-013-9520-0 . hal-01278957

\section{HAL Id: hal-01278957 https://hal.science/hal-01278957}

Submitted on 25 Feb 2016

HAL is a multi-disciplinary open access archive for the deposit and dissemination of scientific research documents, whether they are published or not. The documents may come from teaching and research institutions in France or abroad, or from public or private research centers.
L'archive ouverte pluridisciplinaire HAL, est destinée au dépôt et à la diffusion de documents scientifiques de niveau recherche, publiés ou non, émanant des établissements d'enseignement et de recherche français ou étrangers, des laboratoires publics ou privés. 


\title{
POWER SUMS OF HECKE EIGENVALUES OF MAASS CUSP FORMS
}

\author{
J. WU \& Z. XU
}

\begin{abstract}
In this paper, we establish lower and upper bounds for real positive power sums of Hecke eigenvalues of Maass cusp forms. In particular, we improve a recent result of Holowinsky [5].
\end{abstract}

\section{INTRODUCTION}

Let $f$ be a primitive holomorphic cusp form of even weight $k$ and of level $N$ or a primitive Maass cusp form for the Hecke congruence subgroup $\Gamma_{0}(N)$ with Laplace eigenvalue $\frac{1}{4}+t_{f}^{2}$. Denote by $\lambda_{f}(n)$ its $n$th normalized Fourier coefficient such that $\lambda_{f}(1)=1$. The distribution of these coefficients is one of the most important problems in modular form theory. It is known that they are real and satisfy the Hecke relation. In particular, $\lambda_{f}(n)$ is a real multiplicative function of $n$. For holomorphic forms, the situation is clearer. According to Deligne [3], the Ramanujan-Petersson conjecture

$$
\left|\lambda_{f}(p)\right| \leqslant 2
$$

is true for all primes $p \nmid N$. Thanks to the recent proof of the Sato-Tate conjecture of Barnet-Lamb, Geraghty, Harris \& Taylor [1], the sequence $\left\{\lambda_{f}(p)\right\}_{p \text { primes }}$ is equidistributed on $[-2,2]$ with respect to the Sato-Tate measure. For Maass cusp forms, both conjectures are wide open. In place of (1.1), we have only

$$
\left|\lambda_{f}(p)\right| \leqslant 2 p^{\theta}
$$

for all primes $p$. The best known value $\theta=7 / 64$ is due to Kim \& Sarnak [7]. form

The real positive power sum of Hecke's eigenvalues of primitive holomorphic cusp

$$
S_{f}(x ; r):=\sum_{n \leqslant x}\left|\lambda_{f}(n)\right|^{2 r}
$$

was firstly studied by Rankin ([12], [13]). His method illustrates how to obtain optimally the lower and upper bounds for $S_{f}(x ; r)$ if we only know that the associated Dirichlet series

$$
F_{r}(s):=\sum_{n \geqslant 1}\left|\lambda_{f}(n)\right|^{2 r} n^{-s} \quad(\Re e s>1)
$$

is invertible for $\Re e s \geqslant 1$ (i.e. holomorphic and nonzero for $\Re e s \geqslant 1$ except for $s=1$ ) when $r=1,2$ (The invertibility of these two cases is known by Moreno \& Shahidi

Date: February 25, 2016.

2000 Mathematics Subject Classification. 11F30, 11F66.

Key words and phrases. Fourier coefficients of automorphic forms, Dirichlet series. 
[11]). The key technique of this method is to approximate the function $t \mapsto t^{r}$ optimally by real polynomials of degree 2 on the interval $[0,4]\left(t=\left|\lambda_{f}(p)\right|^{2} \leqslant 4\right)$. Rankin's results are as follows :

$$
x(\log x)^{\delta_{r}^{\mp}} \ll_{f, r} S_{f}(x ; r) \ll_{f, r} x(\log x)^{\delta_{r}^{ \pm}} \quad\left(r \in \mathcal{R}^{\mp}\right)
$$

for $x \geqslant x_{0}(f, r)$, where

$$
\mathcal{R}^{-}:=(0,1] \cup[2, \infty), \quad \mathcal{R}^{+}:=[1,2],
$$

and

$$
\delta_{r}^{-}:=2^{r-1}-1, \quad \delta_{r}^{+}:=\frac{2^{r-1}}{5}\left(2^{r}+3^{2-r}\right)-1 .
$$

The implied constants in (1.5) depend on $f$ and $r$. Since Kim \& Shahidi [9] has proved that $F_{3}(s)$ and $F_{4}(s)$ also are invertible for $\Re e s \geqslant 1$, it is natural to approximate the function $t \mapsto t^{r}$ optimally by real polynomials of degree 4 on the interval $[0,4]$. Based on this idea, Tenenbaum [14] and $\mathrm{Wu}[15]$ found betters bounds.

Now the Sato-Tate conjecture for primitive holomorphic cusp forms has been proved. With the help of this, it is easy to see that

$$
S_{f}(x ; r) \sim C_{r}(f) x(\log x)^{\theta_{r}} \quad(x \rightarrow \infty),
$$

where $C_{r}(f)$ is a positive constant depending on $f, r$, and

$$
\theta_{r}:=\frac{4^{r} \Gamma\left(r+\frac{1}{2}\right)}{\sqrt{\pi} \Gamma(r+2)}-1 .
$$

In the case of Maass cusp forms, the same asymptotic as (1.6) is conjectured to hold with the same exponent $\theta_{r}$, since the corresponding symmetric power $L$-functions are conjectured to the same analytic properties.

Since the conjecture of Ramanujan-Petersson and that of Sato-Tate are both open for Maass cusp forms, it seems interesting to establish analogues of (1.5) for Maass cusp forms. In a private communication with $\mathrm{H}$. Iwaniec, he suggested us considering this problem. The principal difficulty is that we only have (1.2) instead of (1.1). This means that we must approximate the function $t \mapsto t^{r}$ by real polynomials in the unbounded interval $[0, \infty)$ instead of $[0,4]$. In [5], Holowinsky [5, (65)] proposed such an inequality for $r=1 / 2$ :

$$
t^{1 / 2} \leqslant 1+\frac{1}{2}(t-1)-\frac{1}{9}(t-1)^{2}+\frac{1}{36}(t-1)^{3}
$$

valid for all $t \geqslant 0$. Although not stated explicitly, his inequality yields

$$
S_{f}\left(x ; \frac{1}{2}\right) \ll_{f} x(\log x)^{-1 / 12} .
$$

The aim of this paper is to give a general method by developing the ideas in $[13,15]$ and to provide analogues of (1.5) for Maass cusp forms.

Theorem. Let $f$ be a primitive Maass cusp form for $\Gamma_{0}(N)$. Then we have

$$
x(\log x)^{\gamma_{r}^{\mp}} \ll_{f, r} S_{f}(x ; r) \ll_{f, r} x(\log x)^{\gamma_{r}^{ \pm}} \quad\left(r \in \mathfrak{R}^{\mp}\right)
$$

for $x \geqslant x_{0}(f, r)$, where

$$
\mathfrak{R}^{-}:=(0,1] \cup[2,3], \quad \mathfrak{R}^{+}:=[1,2] \cup[3,4]
$$


and

$$
\begin{aligned}
\gamma_{r}^{+}:= \begin{cases}2^{r}-r-1 & \text { if } r \in(0,1] \cup[2,3], \\
3 \cdot 2^{r-3}+2^{2 r-5}-1 & \text { if } r \in(1,2), \\
14 \cdot 4^{r-4}-1 & \text { if } r \in(3,4],\end{cases} \\
\gamma_{r}^{-}:= \begin{cases}\left(3^{r-1}-1\right) / 2 & \text { if } r \in(0,1] \cup[1,2] \cup[3,4], \\
5\left(\frac{14}{5}\right)^{r-3}-1 & \text { if } r \in(2,3) .\end{cases}
\end{aligned}
$$

The implied constants in (1.9) depend on $f$ and $r$. In the lower bound part of (1.9), we need the Ramanujan-Petersson conjecture.

The following table illustrates the difference from the conjectured values (1.6).

\begin{tabular}{|c|c|c|c|c|c|c|c|c|c|}
\hline$r$ & 0 & 0.5 & 1 & 1.5 & 2 & 2.5 & 3 & 3.5 & 4 \\
\hline$\gamma_{r}^{-}$ & -0.3333 & -0.2113 & 0 & 0.3660 & 1 & 1.9880 & 4 & 7.2945 & 13 \\
\hline$\theta_{r}$ & 0 & -0.1512 & 0 & 0.3581 & 1 & 2.1043 & 4 & 7.2781 & 13 \\
\hline$\gamma_{r}^{+}$ & 0 & -0.0857 & 0 & 0.3106 & 1 & 2.1112 & 4 & 6 & 13 \\
\hline
\end{tabular}

The particular case of (1.9) with $r=1 / 2$ and the sign "+" improves Holowinsky's bound (1.8). For comparison, we have

$$
-\frac{1}{12}=-0.0833 \ldots \quad \text { and } \quad \gamma_{1 / 2}^{+}=\sqrt{2}-\frac{3}{2}=-0.0857 \ldots
$$

It is possible to get unconditional lower bounds for $S_{f}(x ; r)$. For example, when $r \geqslant 1$ by using the fact that ${ }^{\dagger}$

$$
S_{f}(x ; j) \asymp_{f, j} x(\log x)^{m_{j}-1} \quad(x \rightarrow \infty, 1 \leqslant j \leqslant 4)
$$

where the $m_{j}$ are given as in (3.3) below, a simple application of the Hölder inequality allows us to deduce

$$
S_{f}(x ; r) \gg_{f, r} x(\log x)^{r\left(m_{j}-1\right) / j} \quad\left(x \geqslant x_{0}(f, r)\right)
$$

for $j \leqslant r \leqslant j+1$ with $j=1,2,3$ or $r \geqslant 4$ with $j=4$. This is better than the lower bound in (1.9) when $r$ is closed to $j$ from its right for $j=1, \ldots, 4$. Similarly when $0<r \leqslant 1$, we have

$$
S_{f}(x ; r) \gg_{f, r} x(\log x)^{-(1-r)}
$$

for $x \geqslant x_{0}(f, r)$, which is weaker than (1.9).

Remark 1. When $r>1$ the functions $\lambda_{f, r}^{ \pm}(n)$ in the lower bound part given in $[13,15]$ are not necessary positive. This will give a problem in the final proof because Tauberian's theorem requires positive coefficients. Our present work corrects them.

\footnotetext{
${ }^{\dagger}$ This is an immediate consequence of the invertibility of $F_{1}(s), F_{2}(s), F_{3}(s)$ and $F_{4}(s)$ for $\Re e s \geqslant 1$.
} 
Acknowledgment. This paper was written when the second author visited l'Institut Élie Cartan de l'Université de Lorraine during the academic year 2011-2012. He would like to thank the institution for the pleasant working conditions. He is supported by China Postdoctoral Science Foundation funded project (2011M501119). We would like to thank the referee for a very careful reading and suggestions.

\section{RANKIN'S IDEA AND REFINEMENT}

In this section, by developing the ideas from $[13,15]$, we shall construct optimal multiplicative functions $\lambda_{f, r}^{ \pm}(n)$ such that

$$
0 \leqslant \lambda_{f, r}^{\mp}(n) \leqslant\left|\lambda_{f}(n)\right|^{2 r} \leqslant \lambda_{f, r}^{ \pm}(n) \quad\left(r \in \mathfrak{R}^{\mp}\right)
$$

for all integers $n \geqslant 1$, and furthermore, their associated Dirichlet series $\Lambda_{f, r}^{ \pm}(s)$ (see (3.1) below) in the half-plane $\Re e s \geqslant 1$ are controlled by $F_{j}(s)$ for $j=1,2,3,4$. Then we can apply the tauberian theorem of Delange [2, Theorem III] (see also $[12$, Lemma 4]) to obtain the asymptotic behaviour of the summatory functions of $\lambda_{f, r}^{ \pm}(n)$.

2.1. Functions of Rankin's type $h_{r,+}\left(t ; \boldsymbol{a}^{+}\right)$with $r \in(0,1) \cup(2,3)$. Consider the function

$$
h_{r,+}\left(t ; \boldsymbol{a}^{+}\right):=t^{r}-a_{1}^{+} t-a_{2}^{+} t^{2}-a_{3}^{+} t^{3} \quad(t \geqslant 0),
$$

where $\boldsymbol{a}^{+}=\boldsymbol{a}^{+}(r)=\left(a_{1}^{+}, a_{2}^{+}, a_{3}^{+}\right)=\left(a_{1}^{+}(r), a_{2}^{+}(r), a_{3}^{+}(r)\right) \in \mathbb{R}^{3}$. Let

$$
0<\kappa<\eta<\infty
$$

be two parameters to be chosen later.

Lemma 2.1. Let $r \in(0,1) \cup(2,3)$. If the function $h_{r,+}\left(t ; \boldsymbol{a}^{+}\right)$defined by $(2.2)$ satisfies

$$
h_{r,+}^{\prime}\left(\kappa ; \boldsymbol{a}^{+}\right)=h_{r,+}^{\prime \prime}\left(\kappa ; \boldsymbol{a}^{+}\right)=h_{r,+}^{\prime}\left(\eta ; \boldsymbol{a}^{+}\right)=0,
$$

then

$$
\left\{\begin{array}{l}
a_{1}^{+}(r)=\frac{r}{\eta-\kappa}\left(\frac{\eta^{r-1} \kappa^{2}+\eta \kappa^{r-1}(\eta-2 \kappa)}{\eta-\kappa}-(r-1) \eta \kappa^{r-1}\right) \\
a_{2}^{+}(r)=\frac{r}{\eta-\kappa}\left(\frac{\kappa^{r}-\kappa \eta^{r-1}}{\eta-\kappa}+\frac{(r-1) \kappa^{r-2}(\eta+\kappa)}{2}\right) \\
a_{3}^{+}(r)=\frac{r}{3(\eta-\kappa)}\left(\frac{\eta^{r-1}-\kappa^{r-1}}{\eta-\kappa}-(r-1) \kappa^{r-2}\right) .
\end{array}\right.
$$

In particular if we take $\ddagger$

$$
(\kappa, \eta)=\left(\kappa_{+}, \eta_{+}\right)=(1,2)
$$

\footnotetext{
$\ddagger$ In Remark 2, we shall explain the reason behind this choice.
} 
then

$$
\left\{\begin{array}{l}
a_{1}^{+}(r)=2 r\left(2^{r-2}-r+1\right), \\
a_{2}^{+}(r)=-r\left(2^{r}-3 r+1\right) / 2, \\
a_{3}^{+}(r)=r\left(2^{r-1}-r\right) / 3 .
\end{array}\right.
$$

Proof. We write (2.3) in the form

$$
\left(\begin{array}{ccc}
1 & 2 \eta & 3 \eta^{2} \\
1 & 2 \kappa & 3 \kappa^{2} \\
0 & 2 & 6 \kappa
\end{array}\right)\left(\begin{array}{c}
a_{1}^{+} \\
a_{2}^{+} \\
a_{3}^{+}
\end{array}\right)=\left(\begin{array}{c}
r \eta^{r-1} \\
r \kappa^{r-1} \\
r(r-1) \kappa^{r-2}
\end{array}\right) .
$$

By resolving this system of linear equations, we can get (2.4).

Lemma 2.2. Let $r \in(0,1) \cup(2,3)$ and let $\boldsymbol{a}^{+}=\left(a_{1}^{+}(r), a_{2}^{+}(r), a_{3}^{+}(r)\right)$ and $h_{r,+}\left(t ; \boldsymbol{a}^{+}\right)$ be defined as in (2.6) and (2.2), respectively. Then we have

$$
t^{r} \leqslant h_{r,+}\left(\eta_{+} ; \boldsymbol{a}^{+}\right)+a_{1}^{+}(r) t+a_{2}^{+}(r) t^{2}+a_{3}^{+}(r) t^{3} \quad(t \geqslant 0) .
$$

Proof. We have

$$
h_{r,+}^{(3)}\left(t ; \boldsymbol{a}^{+}\right)=r(r-1)(r-2) t^{r-3}-6 a_{3}^{+},
$$

so $h_{r,+}^{(3)}\left(t ; \boldsymbol{a}^{+}\right)$has at most one zero for $t>0$ and $h_{r,+}^{(i)}\left(t ; \boldsymbol{a}^{+}\right)$has at most $4-i$ zeros for $t>0(i=2,1,0)$. Since $h_{r,+}^{\prime}\left(\kappa_{+} ; \boldsymbol{a}^{+}\right)=h_{r,+}^{\prime}\left(\eta_{+} ; \boldsymbol{a}^{+}\right)$, it follows that $h_{r,+}^{\prime \prime}\left(\xi_{+} ; \boldsymbol{a}^{+}\right)=0$ for some $\xi_{+} \in\left(\kappa_{+}, \eta_{+}\right)$. Therefore $\kappa_{+}(=1)$ and $\xi_{+}$are the only zeros of $h_{r,+}^{\prime \prime}\left(t ; \boldsymbol{a}^{+}\right)$ in $(0, \infty)$.

Now it is easy to check that

$$
h_{r,+}\left(\kappa_{+} ; \boldsymbol{a}^{+}\right)=\frac{5 r^{2}-9 r+6-2^{r} r}{6}>0 \quad(r \in(0,1) \cup(2,3)) .
$$

Hence for $r \in(0,1) \cup(2,3)$, the function $h_{r,+}\left(t ; \boldsymbol{a}^{+}\right)$is increasing on $\left[0, \eta_{+}\right]$and decreasing on $\left[\eta_{+}, \infty\right)$. Therefore we have

$$
h_{r,+}\left(t ; \boldsymbol{a}^{+}\right) \leqslant h_{r,+}\left(\eta_{+} ; \boldsymbol{a}^{+}\right) \quad(t \geqslant 0),
$$

which is equivalent to the required inequality.

2.2. Functions of Rankin's type $h_{r,+}\left(t ; \boldsymbol{a}^{+}\right)$with $r \in(1,2)$. Consider the function

$$
h_{r,+}\left(t ; \boldsymbol{a}^{+}\right):=t^{r}-a_{2}^{+} t^{2}-a_{3}^{+} t^{3}-a_{4}^{+} t^{4} \quad(t \geqslant 0),
$$

where $\boldsymbol{a}^{+}=\boldsymbol{a}^{+}(r):=\left(a_{2}^{+}, a_{3}^{+}, a_{4}^{+}\right)=\left(a_{2}^{+}(r), a_{3}^{+}(r), a_{4}^{+}(r)\right) \in \mathbb{R}^{3}$. Let

$$
0<\eta<\infty
$$

be a parameter to be chosen later.

Similar to Lemmas 2.1 and 2.2, we have the following results.

Lemma 2.3. Let $r \in(1,2)$. If the function $h_{r,+}\left(t ; \boldsymbol{a}^{+}\right)$defined by $(2.7)$ satisfies

$$
h_{r,+}\left(\eta ; \boldsymbol{a}^{+}\right)=h_{r,+}^{\prime}\left(\eta ; \boldsymbol{a}^{+}\right)=h_{r,+}\left(4 ; \boldsymbol{a}^{+}\right)=0,
$$


then

$$
\left\{\begin{array}{l}
a_{2}^{+}(r)=\frac{4(r-4) \eta^{r-1}+16(3-r) \eta^{r-2}+4^{r-2} \eta^{2}}{(\eta-4)^{2}} \\
a_{3}^{+}(r)=\frac{(4-r) \eta^{r-1}+16(r-2) \eta^{r-3}-2 \cdot 4^{r-2} \eta}{(\eta-4)^{2}} \\
a_{4}^{+}(r)=\frac{(r-3) \eta^{r-2}+4(2-r) \eta^{r-3}+4^{r-2}}{(\eta-4)^{2}}
\end{array}\right.
$$

In particular if we take $\S$

$$
\eta=\eta_{+}=2
$$

then

$$
\left\{\begin{array}{l}
a_{2}^{+}(r)=4^{r-2}+2^{r}-r 2^{r-1} \\
a_{3}^{+}(r)=-4^{r-2}-2^{r-1}+3 r 2^{r-3} \\
a_{4}^{+}(r)=4^{r-3}+2^{r-4}-r 2^{r-4}
\end{array}\right.
$$

Lemma 2.4. Let $r \in(1,2)$ and $\left(a_{2}^{+}, a_{3}^{+}, a_{4}^{+}\right)=\left(a_{2}^{+}(r), a_{3}^{+}(r), a_{4}^{+}(r)\right)$ be given by (2.11). Then we have

$$
t^{r} \geqslant a_{2}^{+} t^{2}+a_{3}^{+} t^{3}+a_{4}^{+} t^{4} \geqslant 0 \quad(0 \leqslant t \leqslant 4) .
$$

Proof. Let $r \in(1,2)$ and let $h_{r,+}\left(t ; \boldsymbol{a}^{+}\right)$be defined as in $(2.7)$ with the choice of $\boldsymbol{a}^{+}=\left(a_{2}^{+}(r), a_{3}^{+}(r), a_{4}^{+}(r)\right)$ given by $(2.11)$. We have

$$
h_{r,+}^{(4)}\left(t ; \boldsymbol{a}^{+}\right)=r(r-1)(r-2)(r-3) t^{r-4}-24 a_{4}^{+},
$$

so $h_{r,+}^{(4)}\left(t ; \boldsymbol{a}^{+}\right)$has at most one zero for $t>0$ and $h_{r,+}^{(i)}\left(t ; \boldsymbol{a}^{+}\right)$has at most $5-i$ zeros for $t>0(i=3,2,1,0)$. Since $h_{r,+}\left(0 ; \boldsymbol{a}^{+}\right)=h_{r,+}\left(\eta_{+} ; \boldsymbol{a}^{+}\right)=h_{r,+}\left(4 ; \boldsymbol{a}^{+}\right)=0$, it follows that $h_{r,+}^{\prime}\left(\xi_{+} ; \boldsymbol{a}^{+}\right)=h_{r,+}^{\prime}\left(\iota_{+} ; \boldsymbol{a}^{+}\right)=0$ for some $\xi_{+} \in\left(0, \eta_{+}\right)$and $\iota_{+} \in\left(\eta_{+}, 4\right)$. Therefore $0, \xi_{+}, \eta_{+}$and $\iota_{+}$are the only zeros of $h_{r,+}^{\prime}\left(t ; \boldsymbol{a}^{+}\right)$in $(0, \infty)$.

Since $r \in(1,2)$, it is easy to verify that

$$
h_{r,+}^{\prime \prime}\left(\eta_{+} ; \boldsymbol{a}^{+}\right)=r(r-1) 2^{r-2}-r 2^{r-1}+2^{r}-2^{2 r-3} \geqslant 0 .
$$

Hence $h_{r,+}\left(t ; \boldsymbol{a}^{+}\right)$takes its minimum values 0 in $[0,4]$ at $0, \eta_{+}$and 4 . Moreover, $h_{r,+}\left(t ; \boldsymbol{a}^{+}\right)$has local maxima values at $\xi_{+}, \iota_{+}$. This shows that $h_{r,+}\left(t ; \boldsymbol{a}^{+}\right) \geqslant 0$ for $0 \leqslant t \leqslant 4$, which implies the first inequality of $(2.12)$.

For $r \in(1,2)$, it is easy to verify that

$$
\left(a_{3}^{+}\right)^{2}-4 a_{2}^{+} a_{4}^{+}=-8^{r-2}+r^{2} 4^{r-3}<0 \quad \text { and } \quad a_{4}^{+}>0 .
$$

Thus we have $a_{2}^{+}+a_{3}^{+} t+a_{4}^{+} t^{2} \geqslant 0$ for all $t \geqslant 0$, which implies the second inequality of (2.12).

\footnotetext{
§In Remark 2, we shall explain the reason behind this choice.
} 
2.3. Functions of Rankin's type $h_{r,-}\left(t ; \boldsymbol{a}^{-}\right)$with $r \in(0,1) \cup(1,2) \cup(3,4)$. Consider the function

$$
h_{r,-}\left(t ; \boldsymbol{a}^{-}\right):=t^{r}-a_{1}^{-} t-a_{2}^{-} t^{2}-a_{3}^{-} t^{3}-a_{4}^{-} t^{4} \quad(t \geqslant 0),
$$

where $\boldsymbol{a}^{-}=\boldsymbol{a}^{-}(r)=\left(a_{1}^{-}, \ldots, a_{4}^{-}\right)=\left(a_{1}^{-}(r), \ldots, a_{4}^{-}(r)\right) \in \mathbb{R}^{4}$. Let

$$
0<\kappa<\eta<\infty
$$

be two parameters to be chosen later.

The next two lemmas are essentially [15, Lemma 2.1] and the part with the sign "-" of [15, Lemma 2.3]. Here we reproduce these lemmas with some small modifications for the convenience of the reader.

Lemma 2.5. Let $r \in(0,1) \cup(1,2) \cup(3,4)$. If the function $h_{r,-}\left(t ; \boldsymbol{a}^{-}\right)$defined by (2.13) satisfies

$$
h_{r,-}^{\prime}\left(\kappa ; \boldsymbol{a}^{-}\right)=h_{r,-}^{\prime}\left(\eta ; \boldsymbol{a}^{-}\right)=h_{r,-}\left(\kappa ; \boldsymbol{a}^{-}\right)=h_{r,-}\left(\eta ; \boldsymbol{a}^{-}\right)=0,
$$

then

$$
a_{j}^{-}(r):=\frac{P_{j}^{-}(\kappa, \eta)-P_{j}^{-}(\eta, \kappa)}{(\kappa-\eta)^{3}}
$$

for $1 \leqslant j \leqslant 4$, where

$$
\begin{aligned}
& P_{1}^{-}(\kappa, \eta):=\{(4-r) \kappa+(r-2) \eta\} \kappa^{r-1} \eta^{2}, \\
& P_{2}^{-}(\kappa, \eta):=\left\{(2 r-8) \kappa^{2}+(1-r) \kappa \eta+(1-r) \eta^{2}\right\} \kappa^{r-2} \eta, \\
& P_{3}^{-}(\kappa, \eta):=\left\{(4-r) \kappa^{2}+(4-r) \kappa \eta+2(r-1) \eta^{2}\right\} \kappa^{r-2}, \\
& P_{4}^{-}(\kappa, \eta):=\{(r-3) \kappa+(1-r) \eta\} \kappa^{r-2} .
\end{aligned}
$$

In particular if we take

$$
(\kappa, \eta)=\left(\kappa_{-}, \eta_{-}\right)=(1,3)
$$

then

$$
\left\{\begin{array}{l}
a_{1}^{-}(r)=\left(-r 3^{r-1}+5 \cdot 3^{r-1}-9 r+9\right) / 4, \\
a_{2}^{-}(r)=\left(7 r 3^{r-2}-34 \cdot 3^{r-2}+15 r-6\right) / 4, \\
a_{3}^{-}(r)=\left(-5 r 3^{r-2}+23 \cdot 3^{r-2}-7 r+1\right) / 4, \\
a_{4}^{-}(r)=\left(r 3^{r-2}-4 \cdot 3^{r-2}+r\right) / 4 .
\end{array}\right.
$$

Lemma 2.6. Let $r \in(0,1) \cup(1,2) \cup(3,4)$ and let $\boldsymbol{a}^{-}:=\left(a_{1}^{-}, \ldots, a_{4}^{-}\right)$be given by (2.16). Then we have

$$
t^{r} \begin{cases}\geqslant a_{1}^{-} t+a_{2}^{-} t^{2}+a_{3}^{-} t^{3}+a_{4}^{-} t^{4} & \text { if } r \in(0,1) \text { and } t \geqslant 0 \\ \leqslant a_{1}^{-} t+a_{2}^{-} t^{2}+a_{3}^{-} t^{3}+a_{4}^{-} t^{4} & \text { if } r \in(1,2) \cup(3,4) \text { and } t \geqslant 0\end{cases}
$$

and

$$
a_{1}^{-} t+a_{2}^{-} t^{2}+a_{3}^{-} t^{3}+a_{4}^{-} t^{4} \geqslant 0 \quad(r \in(0,1), 0 \leqslant t \leqslant 4) .
$$

ฯIn Remark 2, we shall explain the reason behind this choice. 
Proof. Let $r \in(0,1) \cup(1,2) \cup(3,4)$ and let $h_{r,-}\left(t ; \boldsymbol{a}^{-}\right)$be defined as in $(2.13)$ with $\boldsymbol{a}^{-}:=\left(a_{1}^{-}, \ldots, a_{4}^{-}\right)$given by $(2.16)$. We have

$$
h_{r,-}^{(4)}\left(t ; \boldsymbol{a}^{-}\right)=r(r-1)(r-2)(r-3) t^{r-4}-24 a_{4}^{-},
$$

so $h_{r,-}^{(4)}\left(t ; \boldsymbol{a}^{-}\right)$has at most one zero for $t>0$ and $h_{r,-}^{(i)}\left(t ; \boldsymbol{a}^{-}\right)$has at most $5-i$ zeros for $t>0(i=3,2,1,0)$. Since $h_{r,-}\left(0 ; \boldsymbol{a}^{-}\right)=h_{r,-}\left(\kappa_{-} ; \boldsymbol{a}^{-}\right)=h_{r,-}\left(\eta_{-} ; \boldsymbol{a}^{-}\right)=0$, it follows that $h_{r,-}^{\prime}\left(\xi_{-} ; \boldsymbol{a}^{-}\right)=h_{r,-}^{\prime}\left(\iota_{-} ; \boldsymbol{a}^{-}\right)=0$ for some $\xi_{-} \in\left(0, \kappa_{-}\right)$and $\iota_{-} \in\left(\kappa_{-}, \eta_{-}\right)$. Therefore $\xi_{-}, \kappa_{-}, \iota_{-}$and $\eta_{-}$are the only zeros of $h_{r,-}^{\prime}\left(t ; \boldsymbol{a}^{-}\right)$in $(0, \infty)$.

Now

$$
\begin{aligned}
& h_{r,-}^{\prime \prime}\left(\kappa_{-} ; \boldsymbol{a}^{-}\right)=3^{r-2}-(11 / 2) 3^{r-2}+r^{2}-r+3 / 2, \\
& h_{r,-}^{\prime \prime}\left(\eta_{-} ; \boldsymbol{a}^{-}\right)=r^{2} 3^{r-2}-r 3^{r}+(43 / 2) \cdot 3^{r-2}-3 r-3 / 2 .
\end{aligned}
$$

From these, it is easy to verify that

$$
h_{r,-}^{\prime \prime}\left(\kappa_{-} ; \boldsymbol{a}^{-}\right), h_{r,-}^{\prime \prime}\left(\eta_{-} ; \boldsymbol{a}^{-}\right) \begin{cases}>0 & \text { if } r \in(0,1), \\ <0 & \text { if } r \in(1,2) \cup(3,4), \\ =0 & \text { if } r=1,2,3,4 .\end{cases}
$$

Hence $h_{r,-}\left(t ; \boldsymbol{a}^{-}\right)$takes its minimum (maximum, respectively) values 0 in $[0, \infty)$ at $0, \kappa_{-}, \eta_{-}$when $r \in(0,1)\left(r \in(1,2) \cup(3,4)\right.$, respectively). Moreover, $h_{r,-}\left(t ; \boldsymbol{a}^{-}\right)$ has local maxima (minima, respectively) values at $\xi_{-}, \iota_{-}$when $r \in(0,1)(r \in$ $(1,2) \cup(3,4)$, respectively). This shows that

$$
h_{r,-}\left(t ; \boldsymbol{a}^{-}\right) \begin{cases}\geqslant 0 & \text { if } r \in(0,1) \text { and } t \geqslant 0, \\ \leqslant 0 & \text { if } r \in(1,2) \cup(3,4) \text { and } t \geqslant 0,\end{cases}
$$

which is equivalent to $(2.17)$.

Next we prove (2.18). Write

$$
g_{r,-}(t):=a_{1}^{-}+a_{2}^{-} t+a_{3}^{-} t^{2}+a_{4}^{-} t^{3} .
$$

Then

$$
g_{r,-}^{\prime}(t):=a_{2}^{-}+2 a_{3}^{-} t+3 a_{4}^{-} t^{2} .
$$

It is easy to check that

$$
\Delta:=\left(a_{3}^{-}\right)^{2}-3 a_{2}^{-} a_{4}^{-}<0 \quad \text { and } \quad a_{4}^{-}<0
$$

for $r \in(0,1)$. Thus $g_{r,-}^{\prime}(t)<0$ for all $t \in \mathbb{R}$ and $r \in(0,1)$. This shows that when $r \in(0,1)$, the function $t \mapsto g_{r,-}(t)$ is decreasing on $\mathbb{R}$. Since $g_{r,-}(4)=$ $r 3^{r}-3^{r}+3 r+1>0$, we have $g_{r,-}(t)>0$ for $0 \leqslant t \leqslant 4$, which implies immediately $(2.18)$.

2.4. Functions of Rankin's type $h_{r,-}\left(t ; \boldsymbol{a}^{-}\right)$with $r \in(2,3)$. Consider the function

$$
h_{r,+}\left(t ; \boldsymbol{a}^{-}\right):=t^{r}-a_{3}^{-} t^{3}-a_{4}^{-} t^{4} \quad(t \geqslant 0),
$$

where $\boldsymbol{a}^{-}=\boldsymbol{a}^{-}(r):=\left(a_{3}^{-}, a_{4}^{-}\right)=\left(a_{3}^{-}(r), a_{4}^{-}(r)\right) \in \mathbb{R}^{2}$. Let

$$
0<\kappa<\infty
$$

be a parameter to be chosen later. 
Lemma 2.7. Let $r \in(2,3)$. If the function $h_{r,-}\left(t ; \boldsymbol{a}^{-}\right)$defined by $(2.19)$ satisfies

$$
h_{r,-}\left(\eta ; \boldsymbol{a}^{-}\right)=h_{r,-}^{\prime}\left(\eta ; \boldsymbol{a}^{-}\right)=0,
$$

then

$$
\left\{\begin{array}{l}
a_{3}^{-}(r)=(4-r) \eta^{r-3} \\
a_{4}^{-}(r)=-(3-r) \eta^{r-4}
\end{array}\right.
$$

In particular if we take"

$$
\eta=\eta_{-}=\frac{14}{5}
$$

then

$$
\left\{\begin{array}{l}
a_{3}^{-}(r)=(4-r)\left(\frac{14}{5}\right)^{r-3}, \\
a_{4}^{-}(r)=-(3-r)\left(\frac{14}{5}\right)^{r-4} .
\end{array}\right.
$$

Lemma 2.8. Let $r \in(2,3)$ and $\left(a_{3}^{-}, a_{4}^{-}\right)=\left(a_{3}^{-}(r), a_{4}^{-}(r)\right)$ be given by $(2.23)$. Then we have

$$
t^{r} \geqslant a_{3}^{-} t^{3}+a_{4}^{-} t^{4} \geqslant 0 \quad(0 \leqslant t \leqslant 4) .
$$

Proof. Let $r \in(2,3)$ and let $h_{r,-}\left(t ; \boldsymbol{a}^{-}\right)$be defined as in (2.19) with the choice of $\boldsymbol{a}^{-}=\left(a_{3}^{-}(r), a_{4}^{-}(r)\right)$ given by $(2.23)$. We have

$$
h_{r,-}^{(4)}\left(t ; \boldsymbol{a}^{-}\right)=r(r-1)(r-2)(r-3) t^{r-4}-24 a_{4}^{-},
$$

so $h_{r,-}^{(4)}\left(t ; \boldsymbol{a}^{-}\right)$has at most one zero for $t>0$ and $h_{r,-}^{(i)}\left(t ; \boldsymbol{a}^{-}\right)$has at most $5-i$ zeros for $t>0(i=3,2,1,0)$. Since $h_{r,-}\left(0 ; \boldsymbol{a}^{-}\right)=h_{r,-}\left(\eta_{-} ; \boldsymbol{a}^{-}\right)=0$, it follows that $h_{r,-}^{\prime}\left(\xi_{-} ; \boldsymbol{a}^{-}\right)=0$ for some $\xi_{-} \in\left(0, \eta_{-}\right)$. Therefore 0 (double), $\xi_{-}$and $\eta_{-}$are the only zeros of $h_{r,-}^{\prime}\left(t ; \boldsymbol{a}^{-}\right)$in $(0, \infty)$.

Since $r \in(2,3)$, it is easy to verify that

$$
h_{r,-}^{\prime \prime}\left(\eta_{-} ; \boldsymbol{a}^{-}\right)=\left(\frac{14}{5}\right)^{r-2}\left(r^{2}-7 r+12\right) \geqslant 0 .
$$

Hence $h_{r,-}\left(t ; \boldsymbol{a}^{-}\right)$takes its minimum values 0 in $[0, \infty)$ at 0 and $\eta_{-}$. Moreover, $h_{r,-}\left(t ; \boldsymbol{a}^{-}\right)$has a local maximum value at $\xi_{-}$. This shows that $h_{r,-}\left(t ; \boldsymbol{a}^{-}\right) \geqslant 0$ for $t \geqslant 0$, which implies the first inequality of (2.24).

The second is trivial, since $a_{4}^{-}<0$ and $a_{3}^{-}+4 a_{4}^{-}=\left(\frac{14}{5}\right)^{r-3} \frac{3 r-2}{7}>0$.

2.5. Construction of $\lambda_{f, r}^{ \pm}(n)$ and its positivity. We define the multiplicative functions $\lambda_{f, r}^{+}(n)$ by

$$
\lambda_{f, r}^{+}\left(p^{\nu}\right):= \begin{cases}\sum_{0 \leqslant j \leqslant 3} a_{j}^{+}(r) \lambda_{f}(p)^{2 j} & \text { if } \nu=1 \text { and } r \in(0,1) \cup(2,3), \\ \sum_{2 \leqslant j \leqslant 4} a_{j}^{+}(r) \lambda_{f}(p)^{2 j} & \text { if } \nu=1 \text { and } r \in(1,2), \\ 4^{r-4} \lambda_{f}(p)^{8} & \text { if } \nu=1 \text { and } r \in(3,4), \\ 0 & \text { if } \nu \geqslant 2 \text { and } r \in(1,2) \cup(3,4), \\ \left|\lambda_{f}\left(p^{\nu}\right)\right|^{2 r} & \text { if } \nu \geqslant 2 \text { and } r \in(0,1) \cup(2,3),\end{cases}
$$

\footnotetext{
"In Remark 2, we shall explain the reason behind this choice.
} 
where for $r \in(0,1) \cup(2,3), \boldsymbol{a}^{+}(r):=\left(a_{1}^{+}(r), a_{2}^{+}(r), a_{3}^{+}(r)\right)$ is as in (2.6),

$$
a_{0}^{+}(r):=h_{r,+}\left(\eta_{+} ; \boldsymbol{a}^{+}\right)=\eta_{+}^{r}-a_{1}^{+}(r) \eta_{+}-a_{2}^{+}(r) \eta_{+}^{2}-a_{3}^{+}(r) \eta_{+}^{3} \quad\left(\eta_{+}=2\right) ;
$$

and for $r \in(1,2), \boldsymbol{a}^{+}(r):=\left(a_{2}^{+}(r), a_{3}^{+}(r), a_{4}^{+}(r)\right)$ is as in (2.11).

We define the multiplicative function $\lambda_{f, r}^{-}(n)$ by

$$
\lambda_{f, r}^{-}\left(p^{\nu}\right):= \begin{cases}\sum_{1 \leqslant j \leqslant 4} a_{j}^{-}(r) \lambda_{f}(p)^{2 j} & \text { if } \nu=1 \text { and } r \in(0,1) \cup(1,2) \cup(3,4), \\ \sum_{3 \leqslant j \leqslant 4} a_{j}^{-}(r) \lambda_{f}(p)^{2 j} & \text { if } \nu=1 \text { and } r \in(2,3), \\ 0 & \text { if } \nu \geqslant 2 \text { and } r \in(0,1) \cup(2,3), \\ \left|\lambda_{f}\left(p^{\nu}\right)\right|^{2 r} & \text { if } \nu \geqslant 2 \text { and } r \in(1,2) \cup(3,4),\end{cases}
$$

where for $r \in(0,1) \cup(1,2) \cup(3,4), \boldsymbol{a}^{-}(r):=\left(a_{1}^{-}(r), \ldots, a_{4}^{-}(r)\right)$ is as in (2.16); and for $r \in(2,3), \boldsymbol{a}^{-}(r):=\left(a_{3}^{-}(r), a_{4}^{-}(r)\right)$ is as in $(2.23)$.

By applying Lemmas 2.2, 2.4, 2.6 and 2.8 with $t=\left|\lambda_{f}(p)\right|^{2}$, we can deduce that

$$
0 \leqslant \lambda_{f, r}^{\mp}\left(p^{\nu}\right) \leqslant\left|\lambda_{f}\left(p^{\nu}\right)\right|^{2 r} \leqslant \lambda_{f, r}^{ \pm}\left(p^{\nu}\right) \quad\left(r \in \mathfrak{R}^{\mp}\right)
$$

for all primes $p$ and integers $\nu \geqslant 1$, where we need the Ramanujan-Petersson conjecture in the first two inequalities. Obviously (2.28) implies (2.1).

\section{DiRICHLET SERIES ASSOCIATED TO $\lambda_{f, r}^{ \pm}(n)$}

Let $f$ be a primitive Maass cusp form for $\Gamma_{0}(N)$ and let $0<r \leqslant 4$. For $\Re e s>1$, we define

$$
\Lambda_{f, r}^{ \pm}(s):=\sum_{n \geqslant 1} \lambda_{f, r}^{ \pm}(n) n^{-s}
$$

where $\lambda_{f, r}^{ \pm}(n)$ are given as in (2.25) and (2.27). Next we shall study their analytic properties in the half-plane $\Re e s \geqslant 1$ by using the higher order symmetric power $L$-functions $L\left(s, \operatorname{sym}^{m} f\right)$ associated to $f$. (For the definition of $L\left(s, \operatorname{sym}^{m} f\right)$, see $[6$, Chapter 13]) It is known that the function $L\left(s, \operatorname{sym}^{m} f\right)$ is invertible for $\Re e s \geqslant 1$ when $m=2,4,6,8$, due to Gelbart \& Jacquet [4] for $m=2, \operatorname{Kim} \&$ Shahidi $([8,9])$ for $m=4,6,8$.

The next lemma is a particular case of [10, Lemma 7.1]. ** For the absolute convergence of $H_{j}(s)$ below, see [10, Remark 1.7] and its proof at the end of the paper.

Lemma 3.1. Let $f$ be a primitive Maass cusp form for $\Gamma_{0}(N)$ and let $F_{r}(s)$ be defined by (1.4). For $j=1,2,3,4$ and $\Re$ e $s>1$, we have

$$
F_{j}(s)=\zeta(s)^{m_{j}} G_{j}(s) H_{j}(s),
$$

where $\zeta(s)$ is the Riemann $\zeta$-function,

$$
m_{1}:=1, \quad m_{2}:=2, \quad m_{3}:=5, \quad m_{4}:=14,
$$

\footnotetext{
${ }^{* *}$ Since $L\left(s, \operatorname{sym}^{m} f\right)$ with $1 \leqslant j \leqslant 8$ is invertible for $\Re e s \geqslant 1$, the decomposition (3.2) holds for $\Re e s>1$ by analytic continuation.
} 
and

$$
\begin{aligned}
& G_{1}(s):=L\left(s, \operatorname{sym}^{2} f\right) \\
& G_{2}(s):=L\left(s, \operatorname{sym}^{2} f\right)^{3} L\left(s, \operatorname{sym}^{4} f\right), \\
& G_{3}(s):=L\left(s, \operatorname{sym}^{2} f\right)^{9} L\left(s, \operatorname{sym}^{4} f\right)^{5} L\left(s, \operatorname{sym}^{6} f\right), \\
& G_{4}(s):=L\left(s, \operatorname{sym}^{2} f\right)^{34} L\left(s, \operatorname{sym}^{4} f\right)^{20} L\left(s, \operatorname{sym}^{6} f\right)^{7} L\left(s, \operatorname{sym}^{8} f\right)
\end{aligned}
$$

are invertible for $\Re e s \geqslant 1$. Here the function $H_{j}(s)$ admits a Dirichlet series convergent absolutely in $\Re$ e $s>\sigma_{j}$ with $\sigma_{1}=\frac{1}{2}, \sigma_{2}=\frac{53}{96}, \sigma_{3}=\frac{53}{64}, \sigma_{4}=\frac{95}{96}$ and $H_{j}(s) \neq 0$ for $\Re$ e $s=1$.

The next lemma is essentially [15, Lemma 2.5].

Lemma 3.2. Let $f$ be a primitive Maass cusp form for $\Gamma_{0}(N)$ and let $0<r \leqslant 4$. For $\Re$ e $s>1$, we have

$$
\Lambda_{f, r}^{ \pm}(s)=\zeta(s)^{\gamma_{r}^{ \pm}+1} H_{f, r}^{ \pm}(s)
$$

where

$$
\begin{aligned}
& \gamma_{r}^{+}:= \begin{cases}\eta_{+}^{r}+\sum_{1 \leqslant j \leqslant 3}\left(m_{j}-\eta_{+}^{j}\right) a_{j}^{+}(r)-1 & \text { if } r \in(0,1) \cup(2,3), \\
\sum_{2 \leqslant j \leqslant 4} m_{j} a_{j}^{+}(r)-1 & \text { if } r \in(1,2), \\
m_{4} 4^{r-4}-1 & \text { if } r \in(3,4),\end{cases} \\
& \gamma_{r}^{-}:= \begin{cases}\sum_{1 \leqslant j \leqslant 4} m_{j} a_{j}^{-}(r)-1 & \text { if } r \in(0,1) \cup(1,2) \cup(3,4), \\
\sum_{3 \leqslant j \leqslant 4} m_{j} a_{j}^{-}(r)-1 & \text { if } r \in(2,3),\end{cases}
\end{aligned}
$$

and $H_{f, r}^{ \pm}(s)$ is invertible for $\Re$ e $s \geqslant 1$.

Proof. By definition (2.25), for $\Re$ e $s>1$ we can write

$$
\begin{aligned}
\Lambda_{f, r}^{+}(s) & =\prod_{p}\left(1+\sum_{2 \leqslant j \leqslant 4} a_{j}^{+} \lambda_{f}(p)^{2 j} p^{-s}\right) \\
& =\prod_{2 \leqslant j \leqslant 4} F_{j}(s)^{a_{j}^{+}} H_{f, r}^{+}(s)
\end{aligned}
$$

for $r \in(1,2)$, and

$$
\begin{aligned}
\Lambda_{f, r}^{+}(s) & =\prod_{p}\left(1+4^{r-4} \lambda_{f}(p)^{8} p^{-s}\right) \\
& =F_{4}(s)^{4^{r-4}} H_{f, r}^{+}(s)
\end{aligned}
$$

for $r \in(3,4)$, and

$$
\begin{aligned}
\Lambda_{f, r}^{+}(s) & =\prod_{p}\left(1+\sum_{0 \leqslant j \leqslant 3} a_{j}^{+} \lambda_{f}(p)^{2 j} p^{-s}+\sum_{\nu \geqslant 2}\left|\lambda_{f}\left(p^{\nu}\right)\right|^{2 r} p^{-\nu s}\right) \\
& =\prod_{0 \leqslant j \leqslant 3} F_{j}(s)^{a_{j}^{+}} H_{f, r}^{+}(s)
\end{aligned}
$$

for $r \in(0,1) \cup(2,3)$, where $F_{0}(s)=\zeta(s)$ is the Riemann zeta-function and $H_{f, r}^{+}(s)$ is a Dirichlet series absolutely convergent for $\Re e s>\frac{15}{16}$ such that $H_{f, r}^{+}(s) \neq 0$ for 
$\Re e s=1$. Here the constant $\frac{15}{16}$ can be found after some simple calculation with the help of (1.2) and the estimation of Kim \& Shahidi [9] :

$$
\sum_{p \leqslant x} \frac{\lambda_{f}(p)^{8}}{p} \ll \log \log x \quad(x \geqslant 3) .
$$

The part with the "-" sign can be proved similarly.

Remark 2. If we regard $\kappa, \eta$ as parameters, the $\gamma_{r}^{+}=\gamma_{r}^{+}(\kappa, \eta)$ with $r \in(0,1) \cup(2,3)$ and $\gamma_{r}^{-}=\gamma_{r}^{-}(\kappa, \eta)$ with $r \in(0,1) \cup(1,2) \cup(3,4)$ given by (3.5) and (3.6) are functions of these parameters (replacing (2.11) by (2.9) in (3.5) and (2.16) by (2.14) in (3.6), respectively). We choose $\left(\kappa_{ \pm}, \eta_{ \pm}\right)$in $(0, \infty)^{2}$ such that

$$
\frac{\partial \gamma_{r}^{ \pm}(\kappa, \eta)}{\partial \kappa}=0 \quad \text { and } \quad \frac{\partial \gamma_{r}^{ \pm}(\kappa, \eta)}{\partial \eta}=0
$$

which can be done by using a formal calculation via Maple. Their values are given by (2.5) and (2.15), respectively. For these values, we obtain

$$
\begin{aligned}
& \gamma_{r}^{+}=2^{r}-r-1 \quad(r \in(0,1) \cup(2,3)), \\
& \gamma_{r}^{-}=\left(3^{r-1}-1\right) / 2 \quad(r \in(0,1) \cup(1,2) \cup(3,4)) .
\end{aligned}
$$

The others cases can be optimized similarly.

\section{Proof of the Theorem}

In view of Lemma 3.2 and the classical fact on $\zeta(s)$, we can write

$$
\Lambda_{f, r}^{ \pm}(s)=\frac{H_{f, r}^{ \pm}(1)}{(s-1)^{\gamma_{r}^{ \pm}+1}}+g_{f, r}^{ \pm}(s)
$$

in some neighbourhood of $s=1$ with $\Re e s>1$, where $H_{f, r}^{ \pm}(1) \neq 0$ and $g_{f, r}^{ \pm}(s)$ is holomorphic at $s=1$. Thus we can apply Delange's tauberian theorem [2] to deduce

$$
\sum_{n \leqslant x} \lambda_{f, r}^{ \pm}(n) \sim H_{f, r}^{ \pm}(1) x(\log x)^{\gamma_{r}^{ \pm}} \quad(x \rightarrow \infty) .
$$

Now the Theorem follows from (2.1) and (4.1).

\section{On a QUESTION POSED AT AIM}

During the GL(3) workshop at AIM in Palo Alto in 2008, the following question was asked : Let $f$ be a primitive Maass cusp form for $\Gamma_{0}(N)$. Assuming the Ramanujan-Petersson conjecture, find a positive constant $\delta$ such that

$$
\sum_{p \leqslant x} \frac{\left|\lambda_{f}\left(p^{2}\right)\right|}{p} \leqslant(1-\delta) \log \log x+O_{f}(1)
$$

for all $x \geqslant 3$. On the last day of the conference, Holowinsky answered that $\delta=\frac{2}{71}=$ $0.0281 \ldots$ is admissible. His ingredient is the following inequality

$$
t^{1 / 2} \leqslant \frac{69}{71}+\frac{75}{142}(t-1)-\frac{4}{142}\left(t^{2}-3\right)
$$


valid for $0 \leqslant t \leqslant 9$. Obviously our method is also applicable to this problem for establishing lower and upper bounds. For illustration, we can find a better inequality

$$
t^{1 / 2} \leqslant \frac{3+16 \sqrt{3}}{33}+\frac{49 \sqrt{3}-8}{121}(t-1)-\frac{52 \sqrt{3}-48}{1089}\left(t^{2}-3\right)
$$

valid for $0 \leqslant t \leqslant 9$. This implies that $(5.1)$ holds with

$$
\delta=\frac{30-16 \sqrt{3}}{33}=0.0693 \ldots
$$

Also, we can find an inequality

$$
t^{1 / 2} \geqslant \frac{\sqrt{3}}{3}+\frac{\sqrt{3}}{2}(t-1)-\frac{\sqrt{3}}{18}\left(t^{2}-3\right) \geqslant 0
$$

valid for $0 \leqslant t \leqslant 9$. And this implies that

$$
\sum_{p \leqslant x} \frac{\left|\lambda_{f}\left(p^{2}\right)\right|}{p} \geqslant \frac{\sqrt{3}}{3} \log \log x+O_{f}(1) .
$$

\section{REFERENCES}

[1] T. Barnet-Lamb, D. Geraghty, M. Harris \& R. Taylor, A family of Calabi-Yau varieties and potential automorphy. II, Pub. Res. Inst. Math. Sci., 47 (2011), no. 1, 29-98.

[2] H. Delange, Généralisation du théorème de Ikehara, Ann. Sci. Ecole Norm. Sup. 71 (1954), 213-242.

[3] P. Deligne, La conjecture de Weil, I, II, Publ. Math. IHES 48 (1974), 273-308, 52 (1981), 313-428.

[4] S. Gelbart \& H. Jacquet, A relation between automorphic representations of GL(2) and GL(3), Ann. Sci. École Norm. Sup. (4) 11 (1978), no. 4, 471-542.

[5] R. Holowinsky, A sieve method for shifted conveolution sums, Duke Math. J. 146 (2009), no. $3,401-448$.

[6] H. Iwaniec, Topics in Classical Automorphic Forms, Graduate Studies in Mathematics, vol. 17, American Mathematical Society, Providence, Rhode Island, 1997.

[7] H. Kim, Functoriality for the exterior square of $G L_{4}$ and the symmetric fourth of $G L_{2}$, with appendix 1 by D. Ramakrishnan and appendix 2 by H. Kim and P. Sarnak, J. Amer. Math. Soc. 16 (2003), 139-183.

[8] H. H. Kim \& F. Shahidi, Functorial products for $\mathrm{GL}_{2} \times \mathrm{GL}_{3}$ and the symmetric cube for $\mathrm{GL}_{2}$. With an appendix by Colin J. Bushnell and Guy Henniart. Ann. of Math. (2) 155 (2002), no. $3,837-893$.

[9] H. H. Kim \& F. Shahidi, Cuspidality of symmetric powers with applications, Duke Math. J. 112 (2002), no. 1, 177-197.

[10] Y. K. Lau \& G. S. Lü, Sums of Fourier coefficients of cusp forms, The Quarterly Journal of Math. 62 (2011), no. 3, 687-716.

[11] C. J. Moreno \& F. Shahidi, The fourth moment of Ramanujan $\tau$-function, Math. Ann. 266 (1983), no. 2, 233-239.

[12] R. A. Rankin, Sums of powers of cusp form coefficients, Math. Ann. 263 (1983), no. 2, $227-236$.

[13] R. A. Rankin, Sums of powers of cusp form coefficients, II, Math. Ann. 272 (1985), no. 4, $593-600$.

[14] G. Tenenbaum, Remarques sur les valeurs moyennes de fonctions multiplicatives, Enseignement Mathématique (2) 53 (2007), 155-178.

[15] J. Wu, Power sums of Hecke eigenvalues and applications, Acta Arith. 137 (2009), 333-344. 
Jie Wu, School of Mathematics, Shandong University, Jinan, Shandong 250100 , China. CNRS, Institut Élie Cartan de Lorraine, UMR 7502, F-54506 VandeuvreLÈs-NANCY, FRANCE

Current address: Université de Lorraine, Institut Élie Cartan de Lorraine, UMR 7502, F-54506 Vandœuvre-lès-Nancy, France

E-mail address: jie.wu@univ-lorraine.fr

Zhao Xu, School of Mathematics, Shandong University, Jinan, Shandong 250100, CHINA

E-mail address: zxu@sdu.edu.cn 